\author{
Г.В. Буторин ${ }^{1}$, А.Т. Ключников ${ }^{1}$, Е.А. Чабанов ${ }^{1,2}$ \\ ${ }^{1}$ Пермский национальный исследовательский политехнический университет, \\ Пермь, Россия \\ ²Волжский государственный университет водного транспорта (Пермский филиал), \\ Пермь, Россия
}

\title{
ИССЛЕДОВАНИЕ РЕЖИМОВ ПУСКА СИНХРОННОГО ДВИГАТЕЛЯ ПРИ ПОНИЖЕННОЙ ЧАСТОТЕ
}

Традиционно пуск синхронного двигателя осуществляется в асинхронном режиме с обмоткой, замкнутой на добавочное сопротивление. Все способы пуска асинхронного двигателя могут применяться для синхронного двигателя. Способ пуска двигателя зависит от его режима работы и величины момента сопротивления, действующего на валу.

В статье представлены результаты исследований пуска синхронного двигателя при различных параметрах источника питания и моментах сопротивления на валу двигателя. Исследования проводились на моделях, построенных в графической среде имитационного моделирования Simulink пакета прикладных программ для решения задач технических вычислений MatLab. Это программное обеспечение позволяет моделировать и исследовать различные режимы пуска синхронного двигателя при разных величинах питающего напряжения и его частоты.

Было исследовано несколько вариантов пуска синхронного двигателя с возбуждением при различных частотах порядка 2,5-10 Гц. Исследование режимов нагрузки осуществлялось после синхронного пуска двигателя с возбуждением во всем диапазоне частот. Наброс нагрузки производился через отрезок времени 0,2 с. Мощность нагрузки оставалась неизменной для различных частот. Результаты исследования представлены в виде графиков, на основе которых сделаны выводы. Проведенные исследования режима наброса нагрузки при различных частотах показали возможность синхронного пуска в диапазоне частот от 2,5 до 10 Гц. Актуальность работы заключается в том, что ее результаты и сделанные на их основе выводы позволят проектировщикам электрических машин (синхронных двигателей) упростить их проектирование и расчет, а также выявить влияние переходных процессов на исследуемый объект.

Ключевые слова: синхронный двигатель, синхронный пуск на низких частотах, асинхронный пуск, наброс нагрузки, синхронизм, ударный ток, электромагнитный момент, момент сопротивления. 


\section{G.V. Butorin ${ }^{1}$, A.T. Klyuchnikov ${ }^{1}$, E.A. Chabanov ${ }^{1,2}$ \\ ${ }^{1}$ Perm National Research Polytechnic University, Perm, Russian Federation \\ ${ }^{2}$ Volga State University of Water Transport (Perm branch), Russian Federation \\ STUDY OF THE STARTING MODES OF A SYNCHRONOUS ENGINE AT A REDUCED FREQUENCY}

Traditionally, the synchronous engine is started in asynchronous mode with a winding closed to additional resistance. All methods of starting an asynchronous engine can be applied to a synchronous engine. The method of starting the engine depends on its operating mode and on the magnitude of the resistance moment acting on the shaft. The article presents the results of studies on starting a synchronous engine with various parameters of the power source and the resistance moments on the engine shaft. The studies were carried out on models built in the graphical environment of simulation modeling Simulink of application package for solving of technical computing problems MATLAB. This software allows you to simulate and explore various modes of starting a synchronous engine at different values of the supply voltage and its frequency. It was investigated several options for starting a synchronous engine with excitation at various frequencies of the order of $2,5-10 \mathrm{~Hz}$. The study of load conditions was carried out after a synchronous engine start with excitation in the entire frequency range. The load was thrown over a time interval of 0,2 seconds. The load power remained unchanged for different frequencies. The results of the study are presented in the form of graphs, on the basis of which conclusions are drawn. The studies of the load surge mode at various frequencies showed the possibility of synchronous start in the frequency range from 2,5 to $10 \mathrm{~Hz}$. The relevance of the work lies in the fact that its results and conclusions made on their basis will allow designers of electric machines (synchronous engine) to simplify their design and calculation, as well as to identify the effect of transients on the object under study.

Keywords: synchronous engine, synchronous start-up at low frequencies, asynchronous startup, load surge, synchronism, shock current, electromagnetic moment, resistance moment.

При прямом пуске синхронного двигателя в асинхронизированном режиме в цепи статора возникают токи, превосходящие номинальные значения в 7-14 раз, которые поэтому называют ударными. Эти токи вызывают большие электромагнитные нагрузки, которые приводят к ударным механическим перегрузкам не только на двигателе, но и на исполнительных механизмах. Подобные перегрузки способны вывести из строя практически любые потребители механической энергии, питаемые синхронным двигателем. Следовательно, могут увеличиться финансовые затраты на ремонт вышедшего из строя оборудования. Такой пуск синхронного двигателя сопоставим с режимом короткого замыкания, поэтому по обмоткам статора будут протекать довольно большие по величине пусковые токи. По этой причине напряжение реального источника питания из-за возросших тепловых потерь может значительно упасть. Пониженное напряжение электросети может оказывать негативное воздействие на режимы работы другого технологического оборудования, подключенного к ней. Поэтому разработка способов понижения 
пусковых токов синхронных двигателей является весьма актуальной. Системы электропитания, использующие подобные способы, могут повысить безотказность функционирования потребителей электроэнергии, а также обеспечить возможность пуска синхронного двигателя, мощность которого соизмерима с мощностью питающей электросети. Применение таких систем позволяет также увеличить жизненный цикл оборудования, уменьшить вероятность выхода его из строя и возникновения аварийных ситуаций.

Описание исследуемой модели. Для исследования различных режимов после пуска синхронного двигателя (СД) в программной среде MATLAB была собрана модель, схема которой представлена на рис. $1[1,2,3]$.

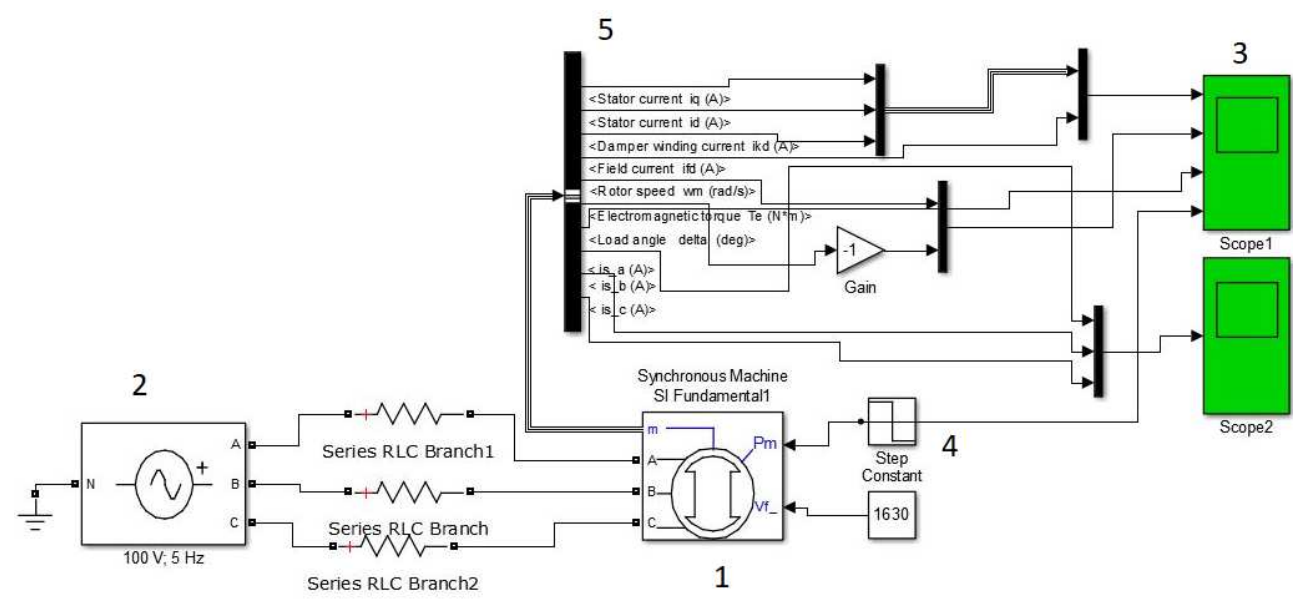

Рис. 1. Схема модели для исследования режимов СД

Схема состоит из трехфазного источника напряжения $(\mathrm{A}, \mathrm{B}, \mathrm{C}) 2$, синхронного двигателя 1 , блоков для установки напряжения возбуждения и момента сопротивления 5 и 4, а также блоков для вывода измеряемых величин 3. Представленная на рис. 1 модель синхронного двигателя является классической. В модели все характеристики и параметры машины представлены в системе СИ [4].

Порты модели А, В и С являются выводами статорной обмотки машины, куда подается входное напряжение. На выходном порту $m$ формируется векторный сигнал, состоящий из 24 элементов.

Источник питающего напряжения является трехфазным, т.е. вырабатывает одновременно систему трех ЭДС с возможностью изменения 
амплитуды, фазы и частоты. По причине неидеальности реального источника электропитания гармонический состав всех трех фаз может также меняться. В нашем случае для СД выбраны базовые величины: напряжение $100 \mathrm{~B}$ с частотой 5 Гц [5]. В синхронном режиме сигнал, соответствующий механической мощности на валу двигателя, подается на входной порт $P m$, также на этот порт можно подавать сигнал в виде скорости $w$ для исследования СД в генераторном режиме. С помощью блока Step задается момент сопротивления соответствующий механической мощности $P m$ на валу двигателя либо скорость $w$. Порт $V f$ служит для подачи напряжения возбуждения на СД, приведенное значение которого задается в блоке Constant. Кроме этого для измерения переменных СД служит блок Bus Selector. Для вывода измеряемых переменных на экран предназначен блок Scope $[1,6]$.

Математическое описание переходных процессов СД. При анализе переходных процессов синхронного двигателя принято использовать систему координат $d$-q. Она описывается двумя системами уравнений $[7,8,9,10,11]$ :

а) система дифференциальных уравнений Парка-Горева:

$$
\left\{\begin{array}{l}
\frac{d \psi_{1 d}}{d t}=u_{d}+\omega \cdot \psi_{1 q}-R_{1} \cdot i_{1 d} \\
\frac{d \psi_{1 q}}{d t}=u_{q}+\omega \cdot \psi_{1 d}-R_{1} \cdot i_{1 q} \\
\frac{d \psi_{f}}{d t}=u_{f}-R_{f} \cdot i_{f} \\
\frac{d \psi_{y d}}{d t}=-R_{y d} \cdot i_{y d} \\
\frac{d \psi_{y q}}{d t}=-R_{y q} \cdot i_{y q} \\
\frac{d \omega}{d t}=\frac{\left(m-m_{c}\right)}{J_{\Sigma}} \\
\frac{d \gamma}{d t}=\omega \\
\frac{d \delta}{d t}=1-\omega .
\end{array}\right.
$$


Напряжение статора определяется проекциями изображающего вектора на координатные оси и описывается уравнениями:

$$
u_{d}=-U_{1} \cdot \sin \delta, u_{q}=U_{1} \cdot \cos \delta
$$

Система алгебраических уравнений, определяющая электромагнитные связи контуров и электромагнитный момент двигателя, описывается уравнениями:

$$
\left\{\begin{array}{l}
d \psi_{1 d}=L_{d} \cdot i_{1 d}+L_{a d}\left(i_{f}+i_{y d}\right) \\
d \psi_{1 q}=L_{q} \cdot i_{1 q}+L_{a q} \cdot i_{y q} \\
\psi_{f}=L_{f} \cdot i_{f}+L_{a d}\left(i_{1 d}+i_{y d}\right) ; \\
\psi_{y d}=L_{y d} \cdot i_{y d}+L_{a d}\left(i_{1 d}+i_{f}\right) ; \\
\psi_{y q}=L_{y q} \cdot i_{y q}+L_{a q} \cdot i_{1 q} \\
m=\psi_{1 d} \cdot i_{1 d}-\psi_{1 q} \cdot i_{1 d} .
\end{array}\right.
$$

Компонентные составляющие токов обмоток двигателя описываются уравнениями:

$$
\begin{gathered}
i_{1 d}=\frac{\psi_{1 d}-\psi_{a d}}{L \delta_{1}} ; i_{1 q}=\frac{\psi_{1 q}-\psi_{a q}}{L \delta_{1}} ; i_{f}=\frac{\psi_{f}-\psi_{a d}}{L \delta_{f}} ; \\
i_{y d}=\frac{\psi_{y d}-\psi_{a d}}{L \delta_{y d}} ; i_{y q}=\frac{\psi_{y q}-\psi_{a q}}{L \delta_{y q}},
\end{gathered}
$$

где $\psi_{a d}, \psi_{a q}$ - компонентные составляющие потокосцепления в воздушном зазоре, которые рассчитываются по формулам:

$$
\psi_{a d}=L_{a d}\left(i_{1 d}+i_{f}+i_{y d}\right) ; \psi_{a q}=L_{a q}\left(i_{1 q}+i_{y q}\right) .
$$

Основным способом пуска синхронного двигателя является асинхронный пуск, при котором обмотка возбуждения двигателя замкнута на пускозащитное сопротивление, величина которого определяется примерно как $R_{\text {пзс }}=10 \cdot R_{f}[12,10]$. Поэтому при асинхронном пуске уравнение для контура обмотки возбуждения имеет вид:

$$
\frac{d \psi_{f}}{d t}=-R_{\text {пзс }} \cdot i_{f} .
$$

Система $d-q$ жестко связана с ротором. Ось $d$ совмещается с продольной осью ротора. Воздушный зазор между статором и ротором 
по осям $d$ и $q$ является неизменным. В рассматриваемой модели в установившемся синхронном режиме составляющие изображенных векторов неизменны во времени. Такой подход упрощает анализ статических характеристик синхронной машины [13, 14].

В программной среде MatLab/Simulink синхронный двигатель описывается так же в системе координат $d-q[1,6]$. На рис. 2 представлена схема замещения классической синхронной машины с демпферной обмоткой. Все параметры могут быть идентифицированы по опытным данным $[15,16,17,18,19]$ и приведены к статорной обмотке $[14,20]$.

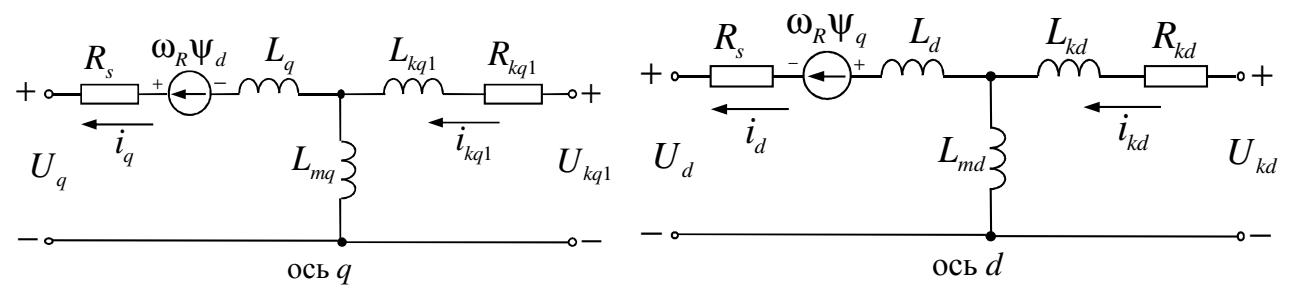

Рис. 2. Схема замещения синхронной машины в системе координат $d-q$

Схема замещения описывается системой уравнений

$$
\left\{\begin{array}{l}
U_{d}=R_{s} i_{d}+\frac{d}{d t} \psi_{d}+\omega_{R} \psi_{q} \\
U_{q}=R_{s} i_{q}+\frac{d}{d t} \psi_{q}-\omega_{R} \psi_{d} \\
U_{f d}=R_{f d} i_{f d}+\frac{d}{d t} \psi_{f d} \\
U_{k d}=R_{k d} i_{k d}+\frac{d}{d t} \psi_{k d} \\
U_{k q 1}=R_{k q 1} i_{k q 1}+\frac{d}{d t} \psi_{k q 1}
\end{array}\right.
$$

где потокосцепления описываются уравнениями:

$$
\begin{aligned}
& \psi_{d}=L_{d} i_{d}+L_{m d}\left(i_{f d}+i_{k d}\right) ; \\
& \psi_{q}=L_{q} i_{q}+L_{m q} i_{k q} ; \\
& \psi_{f d}=L_{f d} i_{f d}+L_{m d}\left(i_{d}+i_{k d}\right) ; \\
& \psi_{k d}=L_{k d} i_{k d}+L_{m d}\left(i_{d}+i_{f d}\right) ; \\
& \psi_{k q 1}=L_{k q 1} i_{k q 1}+L_{m q} i_{q} .
\end{aligned}
$$


Механическая часть синхронной машины описывается следующими уравнениями:

$$
\begin{gathered}
\Delta \omega(t)=\frac{1}{J} \int_{0}^{t}\left(M_{e}-M_{m}\right) d t ; \\
\omega(t)=\Delta \omega(t)+\omega_{0},
\end{gathered}
$$

где $\Delta \omega(t)$ - отклонение текущей частоты вращения ротора от синхронной; $J$ - момент инерции ротора; $M_{m}$ - механический момент; $M_{e}-$ электромагнитный момент; $\omega(t)$ - частота вращения ротора; $\omega_{0}-$ синхронная частота вращения.

Исследование режимов пуска СД. На рис. 3 и 4 представлены графики результатов исследования, полученные для пуска и наброса нагрузки, при напряжении 100 В и с частотой 5 Гц. Момент сопротивления, который задается мощностью, составляет в начале пуска 30 Вт и через 0,2 с меняется на 260 Вт. Исходя из результатов, представленных на рис. 3 и 4, двигатель запускается через 0,12 с, что сопровождается ударными токами $I_{\text {уд }}=41$ А и электромагнитным моментом $M_{\text {эм.уд }}=46,4$ Нм.

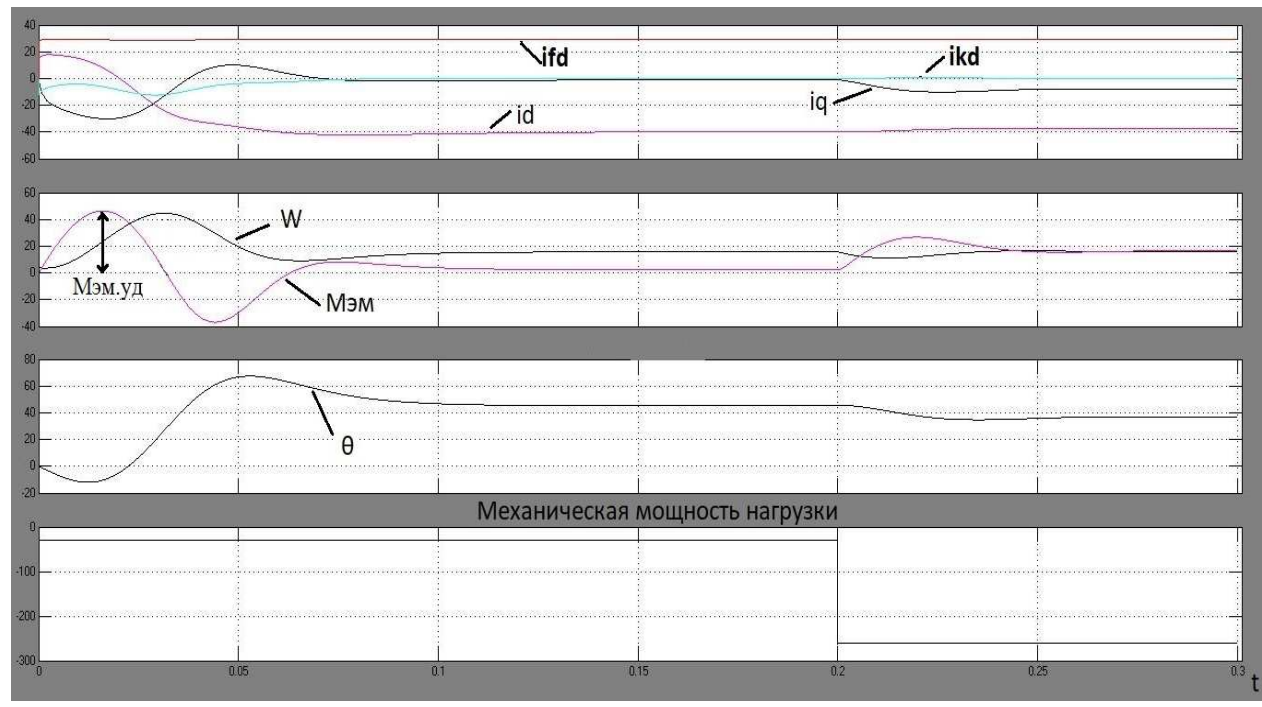

Рис. 3. Результаты исследования пуска СД с последующим набросом нагрузки с частотой питающего напряжения 5 Гц

В интервале времени от 0,12 до 0,2 с наблюдается установившийся процесс, когда ток статора составляет порядка $I_{\mathrm{c}}=39$ А. Электромагнитный момент при этом $M_{\text {эм }}=2,2$ Нм. Скорость в синхронном 
режиме при частоте 5 Гц и составила $\omega=15,5$ рад/с. Ток возбуждения мгновенно возрастает и практически не меняется на всем интервале пуска, так как пуск осуществляется вхолостую и составляет $I_{f}=29$ А. Угол нагрузки равен $\Theta=46,5^{\circ}$.

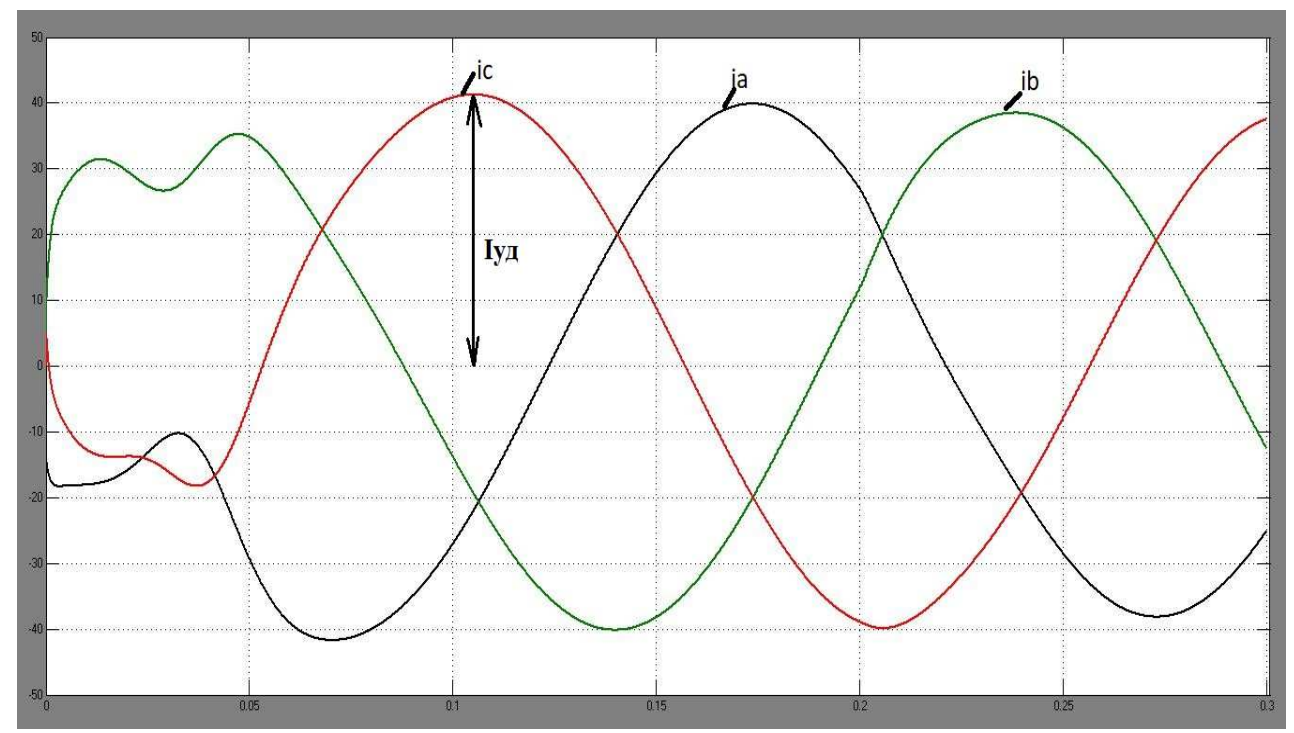

Рис. 4. Графики токов $i_{a}, i_{b}, i_{c}$ статора СД

В момент времени 0,2 с подан момент сопротивления, которому соответствует мощность 260 Вт. С увеличением нагрузки ток статора практически не изменился, а электромагнитный момент во время переходного процесса возрос до $M_{\text {эм }}=25 \mathrm{Hм}$, что соответствует физике процесса. После переходного процесса скорость вернулась до значения $\omega=15,6$ рад/с, а момент $M_{\text {эм }}=17$ Нм. Экспериментальное значение электромагнитной мощности можно вычислить следующим образом:

$$
P_{\text {эм }}=M_{\text {эм }} \cdot \omega=15,6 \cdot 17 \approx 265,5 \text { Вт. }
$$

После наброса нагрузки угол $\Theta=37^{\circ}$.

На рис. 5 и 6 продемонстрированы результаты моделирования пуска СД и наброса на него нагрузки при напряжении питания 100 В и его частоте 3,5 Гц, что составляет половину от первоначальной частоты, использованной в первом опыте. Момент сопротивления подан в то же время, что и для варианта, представленного выше. 


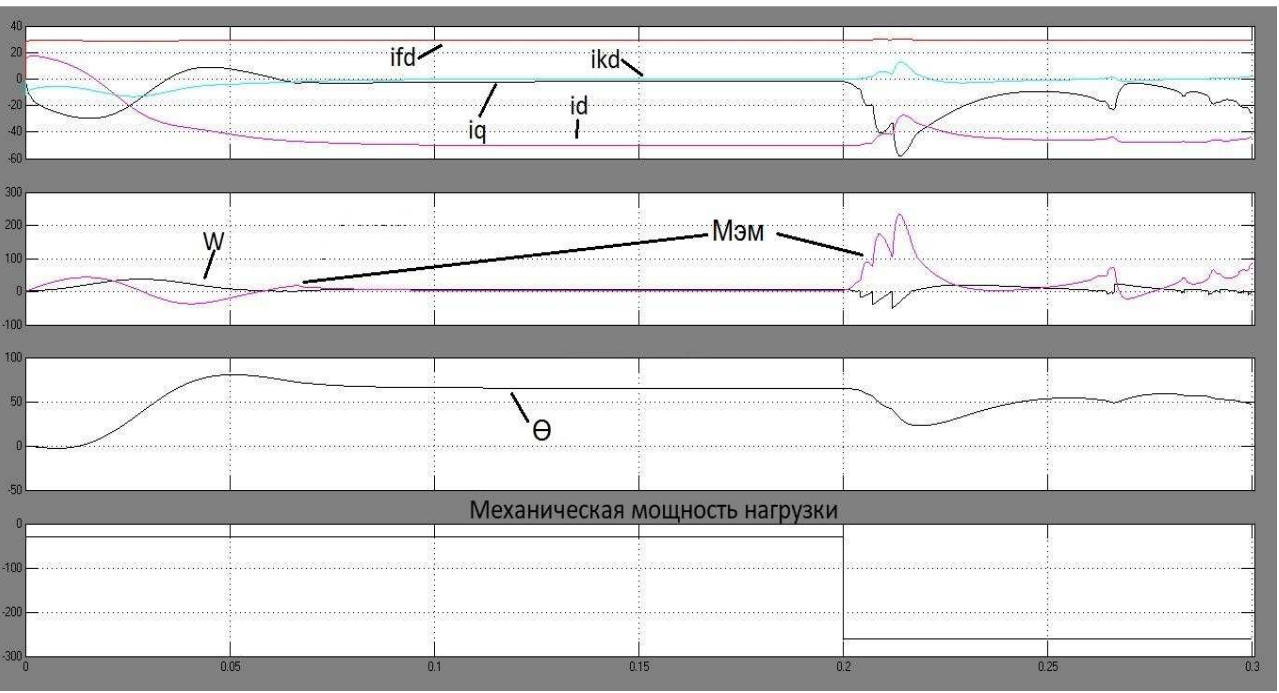

Рис. 5. Результаты исследования пуска СД с последующим набросом нагрузки с частотой питающего напряжения 2,5 Гц

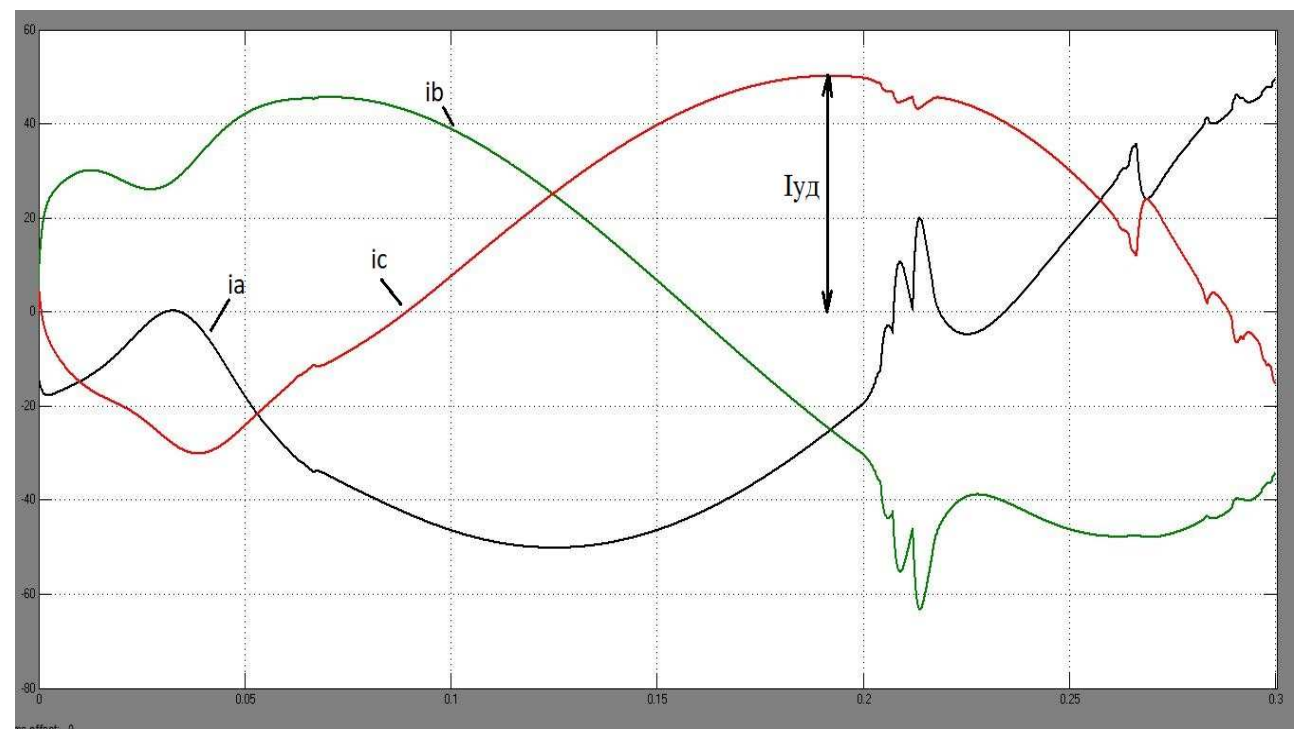

Рис. 6. Графики токов $i_{a}, i_{b}, i_{c}$ статора СД

При уменьшении частоты уменьшается время пуска, следовательно, и время синхронизации должно быть меньше. Как видно из результатов моделирования (см. рис. 5), время пуска, действительно, сократилось до 0,1 с, однако увеличился и ударный ток статора до 50 А. Это объясняется следующим образом: при уменьшении частоты 
индуктивная составляющая сопротивления стала меньше, полное сопротивление так же уменьшилось, следовательно, ток статора увеличился. Ударный электромагнитный момент незначительно изменился до $M_{\text {эм.уд }}=43$ Нм, как и электромагнитный момент на интервале времени до момента наброса нагрузки $M_{\text {эм }}=4$ Нм. Угол нагрузки увеличился до $\Theta=65^{\circ}$ на этом же интервале времени. Ток возбуждения остался практически такой же на всем интервале времени.

Аналогично предыдущему варианту в момент времени $t=0,2$ с был подан момент нагрузки, которому соответствует мощность 260 Вт.

В момент времени 0,2 с, когда был подан повышенный момент сопротивления, двигатель примерно через 0,02 с начал выпадать из синхронизма, на что указывают графики электромагнитного момента и скорости. Следовательно, двигатель запускается при напряжении 100 В и частоте 2,5 Гц, но не способен выдерживать высокие моменты сопротивления на валу.

На рис. 7 и 8 показан вариант нагрузки СД при увеличенной частоте (10 Гц). Момент сопротивления подан так же, как и для вариантов, описанных ранее.

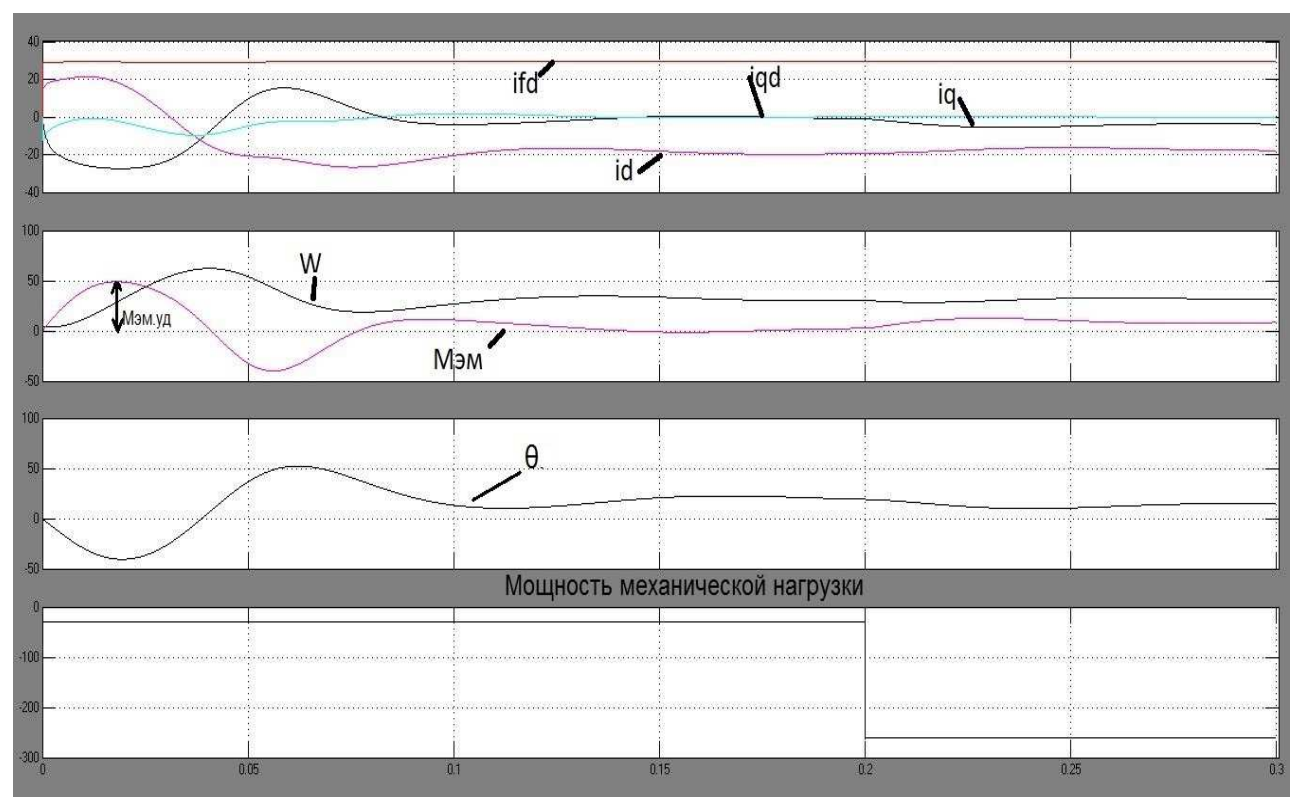

Рис. 7. Результаты исследования пуска СД и с последующим набросом нагрузки с частотой питающего напряжения 10 Гц 


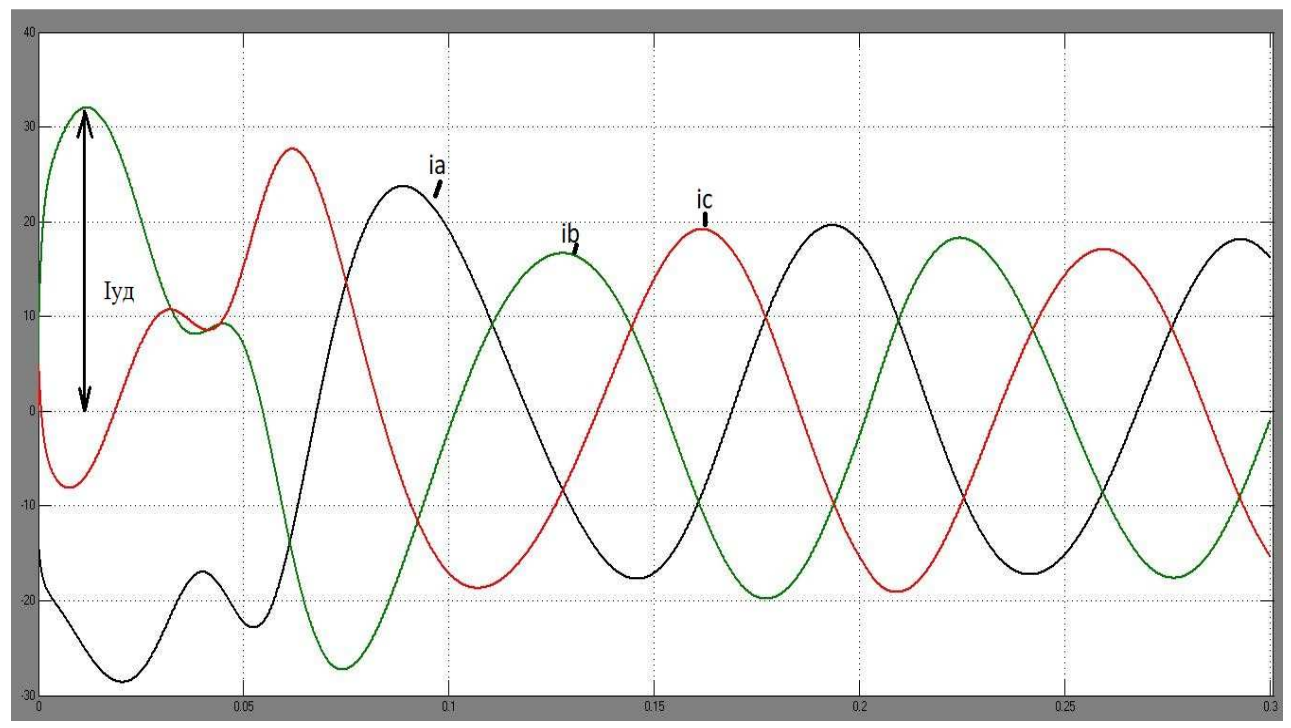

Рис. 8. Графики токов $i_{a}, i_{b}, i_{c}$ статора СД

При увеличении частоты в 2 раза время пуска, как можно наблюдать из результатов моделирования, увеличилось до 0,18 с, но при этом ударный ток статора уменьшился до $I_{\text {уд }}=32 \mathrm{~A}$, а электромагнитный момент достиг значения $M_{\text {эм.уд }}=48,9 \mathrm{Hм}$.

Аналогично предыдущему варианту в момент времени $t=0,2$ с был подан момент нагрузки, которому соответствует мощность 260 Вт.

В момент времени 0,2 с, когда был подан повышенный момент сопротивления, двигатель находился в режиме синхронизма. В дальнейшем (0,3 с) режим синхронизма сохранился, на что указывают графики электромагнитного момента и скорости. При частоте питающего напряжения 10 Гц увеличилось время переходного процесса, однако СД не выпал из синхронизма.

\section{Выводы:}

1. Проведенные исследования режима наброса нагрузки при различных частотах показали возможность синхронного пуска в диапазоне частот от 2,5 до 10 Гц.

2. Наброс нагрузки при постоянной мощности $P m$ на валу сопровождается увеличением момента сопротивления при снижении частоты питающего напряжения. Это закономерно, двигатель при малой частоте питания (2,5 Гц) выпадает из синхронного режима [2]. 
3. При повышенной частоте питающего напряжения (10 Гц) пуск происходит медленнее, чем при частоте, равной 5 Гц, но при этом пуск двигателя и вхождение в синхронизм сопровождаются меньшими ударными токами, что также позволит упростить процесс идентификации параметров синхронной машины [15, 16, 17, 18, 19].

4. Вариант пуска при частоте, равной 5 Гц, оказался оптимальным для проведения дальнейшего исследования. Исходя из представленных результатов, двигатель запускается при напряжении 100 В и частоте 5 Гц с допустимыми ударными значениями токов статора и электромагнитного момента, и при заданных изменениях момента сопротивление не выпадает из синхронизма.

Полученные результаты подтверждают возможность разработки систем и способов плавного пуска синхронных двигателей, применение которых на практике позволит увеличить срок службы электрических машин и других потребителей электроэнергии. Проделанная работа не позволила получить оптимальные параметры плавного пуска синхронных двигателей, поэтому дальнейшие исследования являются крайне перспективными.

\section{Библиографический список}

1. Подольцев А.Д, Бондарь Р.П. Моделирование работы трехфазного линейного синхронного двигателя колебательного движения в пакете MatLab/Simulink // Электротехника и электромеханика. - 2010. № 6. - C. 31-34.

2. Жужгов Н.В., Буторин Г.В., Ключников А.Т. Исследование режимов работы синхронного двигателя // Автоматизированные системы управления и информационные технологии: материалы всероссийской научно-техн. конф.: в 2 т.; Пермь, 17 мая 2018 г. Т. 2. - Пермь: Изд-во Перм. нац. исслед. политехн. ун-та, 2018. - С. 20-27.

3. Черных И.В. Моделирование электротехнических устройств в MatLab, SimPowerSystems и Simulink. - М.: ДМК-Пресс; СПб.: Питер, 2008. - 288 c.

4. Буймов А.А., Кононенко Е.В., Финк А.Ф. Исследование электромеханических переходных процессов тихоходных синхронных реактивных двигателей // Известия Томск. политехн. ун-та. Инжиниринг георесурсов. - Томск, 1972. - Т. 242. - С. 230-233. 
5. Соколовский Г.Г. Электроприводы переменного тока с частотным регулированием: учебник для студ. высш. учеб. завед. - М.: Акадения, 2006. - 272 с.

6. Дьяков В.П. Simulink 5/6/7: самоучитель. - М.: ДМК-Пресс, 2008. - $784 \mathrm{c}$.

7. Фираго Б.И., Александровский С.В. Исследование переходных процессов частотно-регулируемого синхронного электропривода // Известия высших учебных заведений и энергетических объединений СНГ. Энергетика. - 2016. - Т. 59. - № 6. - С. 507-518.

8. Копылов И.П. Математическое моделирование электрических машин. - М.: Высшая школа, 1987. - 248 с.

9. Сипайлов Г.А., Кононенко Е.В., Хорьков К.А. Электрические машины. - М.: Высшая школа, 1987. - 287 с.

10. Вольдек А.И., Попов В.В. Электрические машины. Машины переменного тока. - СПб., 2010. - 352 с.

11. Сравнение методов расчёта электромагнитных процессов на примере цилиндрического линейного вентильного двигателя / Д.А. Чирков, А.Т. Ключников, А.Д. Коротаев, Э.О. Тимашев // Вестник Пермского национального исследовательского политехнического университета. Электротехника, информационные технологии, системы управления. 2018. - № 28. - С. 76-91.

12. Макаричев Ю.А., Овсянников В.Н. Синхронные машины: учеб. пособ. - Самара: Изд-во Самар. гос. техн. ун-та, 2010. - 156 с.

13. Субботина В.А., Тюленев M.E. Simulink-модель для исследования пуска синхронного двигателя при пониженном напряжении // Вестник Пермского национального исследовательского политехнического университета. Электротехника, информационные технологии, системы управления. - 2014. - № 11. - С. 102-109.

14. Осин И.Л., Антонов М.В. Устройство и производство электрических машин малой мощности: учеб. пособие для СПТУ. - М.: Высшая школа, 1988. - 215 с.

15. Судаков А.И., Чабанов Е.А., Шулаков Н.В. Новые подходы к идентификации переходных процессов синхронных машин в опытах внезапного симметричного короткого замыкания вероятностностатистическими методами // Интеллектуальные системы в производстве. -2013 . - № 2(22). - С. 207-213. 
16. Чабанов Е.А., Судаков А.И., Шулаков Н.В. Новые подходы к получению исходной информации и методам идентификации переходных процессов мощных синхронных машин // Вестник Пермского национального исследовательского политехнического университета. Электротехника, информационные технологии, системы управления. 2013. - № 8. - С. 114-127.

17. Судаков А.И., Чабанов Е.А. Высокоточная и достоверная идентификация переходных процессов мощных синхронных машин вероятностно-статистическими методами // Электротехника. - 2015. - № 11. C. 21-27; Sudakov A.I., Chabanov E.A. Precise and reliable identification of the transient processes of a powerful synchronous machine by probabilistic statistical methods // Russian Electrical Engineering. - 2015. - Vol. 86, № 11. - P. 640-645.

18. Судаков А.И., Чабанов Е.А., Каменских И.А. Развитие вероятностно-статистических методов идентификации зашумлённых переходных процессов синхронных машин // Электротехника. - 2017. - № 11. C. 18-24; Sudakov A.I., Chabanov E.A., Kamenskikh I.A. The Development of Probabilistic and Statistical Methods for Identification of Noisy Transient Processes of Synchronous Machines // Russian Electrical Engineering. 2017. - Vol. 88, № 11. - P. 714-719.

19. Sudakov A.I., Chabanov E.A., Shulakov N.V. Novel approaches to analysis of transition processes identification error by probability-statistical methods during sudden symmetric short-circuit tests of synchronous machines // Acta Technica CSAV (Ceskoslovensk Akademie Ved). - 2013. Vol. 58, № 4. - P. 381-392.

20. Проектирование электрических машин: учебник для вузов / И.П. Копылов, Б.К. Клоков, В.П. Морозкин, Б.Ф. Токарев; под ред. И.П. Копылова. - 4-е изд., перераб. и доп. - М.: Юрайт, 2011. - 767 с.

\section{References}

1. Podol'tsev A.D, Bondar' R.P. Modelirovanie raboty trekhfaznogo lineinogo sinkhronnogo dvigatelia kolebatel'nogo dvizheniia $\mathrm{v}$ pakete MatLab/Simulink [Modeling the operation of a three-phase linear synchronous engine of oscillatory motion in the package MatLab/Simulink]. Elektrotekhnika i elektromekhanika, 2010, no. 6, pp. 31-34. 
2. Zhuzhgov N.V., Butorin G.V., Kliuchnikov A.T. Issledovanie rezhimov raboty sinkhronnogo dvigatelia [The study of the synchronous engine operating modes]. Avtomatizirovannye sistemy upravleniia $i$ informatsionnye tekhnologii: materialy vserossiiskoi nauchno-tekhntekhnicheskoi konferentsii, Perm, 17 May 2018. Tom 2. Perm: Permskii natsional'nyi issledovatel'skii politekhnicheskii universitet, 2018, pp. 20-27.

3. Chernykh I.V. Modelirovanie elektrotekhnicheskikh ustroistv v MatLab, SimPowerSystems i Simulink [Modeling of electrical devices in MatLab, SimPowerSystems and Simulink]. Moscow: DMK-Press; Saint Petersburg: Piter, 2008. 288 p.

4. Buimov A.A., Kononenko E.V., Fink A.F. Issledovanie elektromekhanicheskikh perekhodnykh protsessov tikhokhodnykh sinkhronnykh reaktivnykh dvigatelei [The study of electromechanical transients of lowspeed synchronous jet engines]. Izvestiia Tomskogo politekhnicheskogo universiteta. Inzhiniring georesursov. Tomsk, 1972, vol. 242, pp. 230-233.

5. Sokolovskii G.G. Elektroprivody peremennogo toka s chastotnym regulirovaniem [Electric drives of alternating current with frequency regulation]. Moscow: Akadeniia, 2006. 272 p.

6. D'iakov V.P. Simulink 5/6/7: samouchitel' [Simulink 5/6/7: Selfinstruction]. Moscow: DMK-Press, 2008. 784 p.

7. Firago B.I., Aleksandrovskii S.V. Issledovanie perekhodnykh protsessov chastotno-reguliruemogo sinkhronnogo elektroprivoda [The study of transients of a frequency-controlled synchronous electric drive]. Izvestiia vysshikh uchebnykh zavedenii $i$ energeticheskikh ob"edinenii SNG Energetika, 2016, vol. 59, no. 6, pp. 507-518.

8. Kopylov I.P. Matematicheskoe modelirovanie elektricheskikh mashin [Mathematical modeling of electrical machines]. Moscow: Vysshaia shkola, 1987. 248 p.

9. Sipailov G.A., Kononenko E.V., Khor'kov K.A. Elektricheskie mashiny [Electric machines]. Moscow: Vysshaia shkola, 1987. 287 p.

10. Vol'dek A.I., Popov V.V. Elektricheskie mashiny. Mashiny peremennogo toka [Electric machines. AC machines]. Saint Petersburg, 2010. 352 p.

11. Chirkov D.A., Kliuchnikov A.T., Korotaev A.D., Timashev E.O. Sravnenie metodov rascheta elektromagnitnykh protsessov na primere tsilindricheskogo lineinogo ventil'nogo dvigatelia [Comparison of methods for calculating electromagnetic processes on the example of a cylindrical 
linear valve motor]. Vestnik Permskogo natsional'nogo issledovatel'skogo politekhnicheskogo universiteta. Elektrotekhnika, informatsionnye technologii, sistemy upravleniia, 2018, no. 28, pp. 76-91.

12. Makarichev Iu.A., Ovsiannikov V.N. Sinkhronnye mashiny [Synchronous machines]. Samara: Samarskii gosudarstvennyi tekhnicheskii universitet, 2010. $156 \mathrm{p}$.

13. Subbotina V.A., Tiulenev M.E. Simulink-model' dlia issledovaniia puska sinkhronnogo dvigatelia pri ponizhennom napriazhenii [Simulinkmodel for the study of starting a synchronous engine at low voltage]. Vestnik Permskogo natsional'nogo issledovatel'skogo politekhnicheskogo universiteta. Elektrotekhnika, informatsionnye tekhnologii, sistemy upravleniia, 2014, no. 11, pp. 102-109.

14. Osin I.L., Antonov M.V. Ustroistvo i proizvodstvo elektricheskikh mashin maloi moshchnosti [The device and production of low-power electric machines]. Moscow: Vysshaia shkola, 1988. 215 p.

15. Sudakov A.I., Chabanov E.A., Shulakov N.V. Novye podkhody k identifikatsii perekhodnykh protsessov sinkhronnykh mashin $\mathrm{v}$ opytakh vnezapnogo simmetrichnogo korotkogo zamykaniia veroiatnostnostatisticheskimi metodami [New approaches to the identification of transients of synchronous machines in experiments of a sudden symmetric short circuit by probabilistic-statistical methods]. Intellektual'nye sistemy $v$ proizvodstve, 2013, no. 2(22), pp. 207-213.

16. Chabanov E.A., Sudakov A.I., Shulakov N.V. Novye podkhody k polucheniiu iskhodnoi informatsii i metodam identifikatsii perekhodnykh protsessov moshchnykh sinkhronnykh mashin [New approaches to obtaining initial information and methods for identifying transients of powerful synchronous machines]. Vestnik Permskogo natsional'nogo issledovatel'skogo politekhnicheskogo universiteta. Elektrotekhnika, informatsionnye tekhnologii, sistemy upravleniia, 2013, no. 8, pp. 114-127.

17. Sudakov A.I., Chabanov E.A. Vysokotochnaia i dostovernaia identifikatsiia perekhodnykh protsessov moshchnykh sinkhronnykh mashin veroiatnostno-statisticheskimi metodami [Precise and reliable identification of the transient processes of a powerful synchronous machine by probabilistic statistical methods]. Elektrotekhnika, 2015, no. 11, pp. 21-27; Sudakov A.I., Chabanov E.A. Precise and reliable identification of the transient processes of a powerful synchronous machine by probabilistic statistical methods. Russian Electrical Engineering, 2015, vol. 86, no. 11, pp. 640-645. 
18. Sudakov A.I., Chabanov E.A., Kamenskikh I.A. Razvitie veroiatnostno-statisticheskikh metodov identifikatsii zashumlennykh perekhodnykh protsessov sinkhronnykh mashin [The development of probabilistic and statistical methods for identification of noisy transient processes of synchronous machines]. Elektrotekhnika, 2017, no. 11, pp. 18-24; Sudakov A.I., Chabanov E.A., Kamenskikh I.A. The Development of Probabilistic and Statistical Methods for Identification of Noisy Transient Processes of Synchronous Machines. Russian Electrical Engineering, 2017, vol. 88, no. 11, pp. 714-719.

19. Sudakov A.I., Chabanov E.A., Shulakov N.V. Novel approaches to analysis of transition processes identification error by probability-statistical methods during sudden symmetric short-circuit tests of synchronous machines. Acta Technica CSAV (Ceskoslovensk Akademie Ved), 2013, vol. 58, no. 4, pp. 381-392.

20. Kopylov I.P., Klokov B.K., Morozkin V.P., Tokarev B.F. Proektirovanie elektricheskikh mashin [Design of electrical machines]. Ed. I.P. Kopylov. 4nd ed. Moscow: Iurait, 2011. 767 p.

\section{Сведения об авторах}

Буторин Георгий Васильевич (Пермь, Россия) - студент Пермского национального исследовательского политехнического университета (614990, Пермь, Комсомольский пр., 29, e-mail: zhora20091@yandex.ru).

Ключников Анатолий Терентьевич (Пермь, Россия) - кандидат технических наук, доцент кафедры «Электротехника и электромеханика» Пермского национального исследовательского политехнического университета (614990, Пермь, Комсомольский пр., 29, e-mail: aklu2011@pstu.ru).

Чабанов Евгений Александрович (Пермь, Россия) - кандидат технических наук, доцент кафедры «Электротехника и электромеханика» Пермского национального исследовательского политехнического университета (614990, Пермь, Комсомольский пр., 29); доцент кафедры «Технические дисциплины» Пермского филиала Волжского государственного университета водного транспорта (614060, Пермь, Бульвар Гагарина, 33, e-mail: ceapb@mail.ru). 


\section{About the authors}

Butorin Georgii Vasilyevich (Perm, Russian Federation) is a Student Perm National Research Polytechnic University (614990, Perm, 29, Komsomolsky pr., e-mail: zhora20091@yandex.ru).

Klyuchnikov Anatolii Terentyevich (Perm, Russian Federation) is a Ph.D. in Technical Sciences, Associate Professor of Department of Electrical Engineering and Electromechanics of Perm National Research Polytechnic University (614990, Perm, 29, Komsomolsky pr., e-mail: aklu2011@pstu.ru).

Chabanov Evgenii Aleksandrovich (Perm, Russian Federation) is a Ph.D. in Technical Sciences, Associate Professor of Department of Electrical Engineering and Electromechanics Perm National Research Polytechnic University (614990, Perm, 29, Komsomolsky pr.); Associate Professor of Department of Technical Disciplines Volga State University of Water Transport (Perm branch) (614060, Perm, 33, Gagarina bulvar, e-mail: ceapb@mail.ru).

Получено: 17.07.2019 